\title{
Differential Prognostic Value of Metabolic Heterogeneity of Primary Tumor and Metastatic Lymph Nodes in Patients with Pharyngeal Cancer
}

\author{
JEON YEOB JANG ${ }^{1,2^{*}}$, KYOUNG JUNE PAK $^{2,3^{*}}$, KEUN-IK YI $^{1}$, YONG KAN KI ${ }^{4}$, \\ WON TAEK KIM ${ }^{4}$, HYOJEONG KIM ${ }^{5}$, YOUNG JIN CHOI ${ }^{5}$, SEONG JANG KIM ${ }^{6}$, \\ IN JOO KIM ${ }^{2,3}$, SOO-GEUN WANG ${ }^{1,2}$ and YOUNG MI SEOL ${ }^{2,5}$ \\ ${ }^{1}$ Department of Otorhinolaryngology-Head and Neck Surgery, Pusan National University Hospital, \\ Pusan National University School of Medicine, Busan, Republic of Korea; \\ ${ }^{2}$ Biomedical Research Institute, Pusan National University Hospital, Busan, Republic of Korea; \\ ${ }^{3}$ Department of Nuclear Medicine, Pusan National University Hospital, \\ Pusan National University School of Medicine, Busan, Republic of Korea; \\ ${ }^{4}$ Department of Radiation Oncology, Pusan National University Hospital, \\ Pusan National University School of Medicine, Busan, Republic of Korea; \\ ${ }^{5}$ Department of Internal Medicine, Pusan National University Hospital, \\ Pusan National University School of Medicine, Busan, Republic of Korea; \\ ${ }^{6}$ Department of Nuclear Medicine and Research Institute for Convergence of Biomedical Science and Technology, \\ Pusan National University Yangsan Hospital, Yangsan, Republic of Korea
}

\begin{abstract}
Background/Aim: We aimed to explore the prognostic value of metabolic heterogeneity of ${ }^{18}$ F-FDG uptake in chemoradiotherapy-treated pharyngeal cancer patients. Patients and Methods: This study included 52 consecutive patients with pharyngeal cancer who underwent ${ }^{18} F-F D G$ $P E T / C T$ before definitive chemoradiotherapy. The heterogeneity factor $(H F)$ was defined as the derivative (dV/dT) of a volumethreshold function for primary tumors and metastatic lymph nodes. The relationships between clinical parameters and HFs of primary tumors $(\mathrm{pHF})$ and metastatic lymph nodes $(\mathrm{nHF})$ were analyzed. Results: The $p H F$ (range=-1.367 - -0.027; median=-0.152) was significantly correlated with the maximum standardized uptake value, metabolic tumor volume, and total lesion glycolysis. Induction chemotherapy response was not correlated with $\mathrm{HF}$, whereas response to radiotherapy was
\end{abstract}

\footnotetext{
*These Authors contributed equally to this study.

Correspondence to: Young Mi Seol, MD, Ph.D., Department of Internal Medicine, Biomedical Research Institute, Pusan National University Hospital, Pusan National University School of Medicine, 179 Gudeok-ro, Seo-gu, Busan 602-739, Republic of Korea. Tel: +82 512407825, Fax: +82 512407431, e-mail: seol2100@hanmail.net
}

Key Words: Metabolic heterogeneity, PET, pharyngeal cancer, chemoradiotherapy. significantly better in patients with high pHF (low heterogeneity). Consistently, the 2-year locoregional recurrencefree survival was significantly better in patients with high $\mathrm{pHF}$ (82.9\% for $\mathrm{pHF}>-0.152$ vs. $30.5 \%$ for $\mathrm{pHF}<-0.152$, log-rank $p=0.009)$. The $n H F$ (range $=-1.067-0.039 ;$ median $=-0.160$ ) was not correlated with response to radiotherapy and locoregional recurrences. Conclusion: $\mathrm{pHF}$, but not $n \mathrm{HF}$, was a significant predictor of response to radiotherapy and locoregional recurrence in pharyngeal cancer. Thus, HF use can prevent unnecessary treatment and surgical delays.

Pharyngeal squamous cell carcinoma (SCC) patients have a poor overall prognosis with a high likelihood of recurrence at the primary site and metastatic involvement of the cervical lymph nodes (LNs) (1). Different primary and metastatic nodal characteristics may result in different responsiveness to treatment (2). Therefore, it is important to identify patients on the basis of their treatment responsiveness prior to definitive chemoradiation (CRT) and induction chemotherapy. Patients with pathological complete responses would have favorable oncological outcomes, and identification of patients who are likely to have poor responses could prevent unnecessary chemotherapy, radiation, and delays in surgery. Data suggest that traditional risk stratification variables, such as T-category or N-category, have limited value as prognostic and predictive indicators of treatment response or survival (3). Diverse clinical and molecular predictors of treatment 
responsiveness have been investigated $(4,5)$, but no clear consensus currently exists about reliable markers for tumor response to CRT and induction chemotherapy.

In pharyngeal cancer patients, ${ }^{18} \mathrm{~F}$-fluorodeoxyglucose positron emission tomography/computed tomography $\left({ }^{18} \mathrm{~F}-\right.$ FDG PET/CT) has been used widely for staging work-ups, and many ongoing studies have used PET/CT to predict patient prognosis. The most commonly used PET parameter to indicate the degree of ${ }^{18} \mathrm{~F}$-FDG uptake is the standardized uptake value (SUV), which is an independent prognostic factor in head and neck cancer $(6,7)$. Recently, volumetric PET parameters such as the metabolic tumor volume and total lesion glycolysis have emerged as novel prognostic factors that may improve risk stratification and the planning of individualized cancer treatment protocols in patients with pharyngeal cancer (8). Metabolic tumor volume and total lesion glycolysis are also known to be predictive factors for prognosis in other head and neck cancers (9). However, evaluations of these parameters have yielded conflicting results.

Intratumoral heterogeneity has been shown to be a strong indicator of malignant transformation and different characteristics of tumors (10-12). Therefore, the pattern of intratumoral ${ }^{18} \mathrm{~F}$-FDG uptake on PET may represent a similarly useful parameter (13). A recent study revealed that the intratumoral heterogeneity of ${ }^{18} \mathrm{~F}$-FDG uptake in nasopharyngeal and rectal cancer was significantly correlated with tumor aggressiveness and was associated with various outcome measures $(14,15)$. To the best of our knowledge, no study has evaluated the predictive value of the intratumoral heterogeneity of ${ }^{18} \mathrm{~F}$-FDG uptake of primary and/or nodal sites in pharyngeal cancer. Therefore, we investigated the relationship between tumor heterogeneity and various clinical and PET parameters of primary and/or nodal sites and then evaluated the predictive value of heterogeneity factors in locally advanced pharyngeal cancer.

\section{Patients and Methods}

Patients. From January 2012 to December 2014, 52 patients with pharyngeal cancer who were treated with definitive CRT and/or induction chemotherapy were enrolled in this study. Criteria for eligibility also included measurable, histologically-confirmed squamous cell carcinoma of the nasopharynx, oropharynx, or hypopharynx without any evidence of distant metastasis; age between 20 and 80 years; and Eastern Cooperative Oncology Group (ECOG) performance status of 0-2. The patients had received no prior chemotherapy, radiotherapy, or surgery. This study protocol was approved by the Institutional Review Board and the requirement of obtaining informed consent from patients was waived owing to the retrospective medical record review. Patient follow-up lasted until death or until the cut-off date of April 30, 2015. The median followup interval was 39 months (range=5-54 months).

Follow-up studies and evaluation of treatment response. All patients underwent contrast-enhanced neck CT and ${ }^{18}$ F-FDG PET/CT to evaluate the initial disease stage. Additionally, neck ultrasonography and neck magnetic resonance imaging (MRI) were performed if clinically indicated. All patients were followed up for 4-6 weeks after CRT treatment in the outpatient clinic. Further follow up was performed at 3-month intervals for the first and second years, at 6month intervals for the third year, and yearly for the fourth and fifth years. Locoregioanl recurrence of head and neck cancer after CRT was defined as clinical and/or radiological evidence of persisting or recurrent disease at the primary site or within the neck LNs. Metastatic disease was determined using CT.

Definitive CRT. All patients underwent three-dimensional conformal treatment planning using CT scan simulation. Radiation was administered 5 days per week in daily fractions of 1.8-2 Gy, and the total dose to the primary tumor was 70-74 Gy. Intravenous chemotherapy $\left(40 \mathrm{mg} / \mathrm{m}^{2}\right.$ cisplatin) was administered once a week during radiotherapy.

Protocol for ${ }^{18} F-F D G$ PET/CT. Standard patient preparation included at least $8 \mathrm{~h}$ fasting and a serum glucose level of less than $120 \mathrm{mg} / \mathrm{dl}$ before ${ }^{18} \mathrm{~F}-\mathrm{FDG}$ administration. PET/CT imaging was performed $60 \mathrm{~min}$ after injection of $370 \mathrm{MBq}$ of $18 \mathrm{~F}-\mathrm{FDG}$. Patients were hydrated with $500 \mathrm{ml}$ of water per orally before the PET/CT imaging. At $60 \mathrm{~min}$ after administration of $18 \mathrm{~F}-\mathrm{FDG}$, a CT scan was performed first for attenuation correction, followed by an emission scan from the skull base to the proximal thighs. PET images were reconstructed using an iterative algorithm (ordered-subset expectation maximization) with an image matrix size of $256 \times 256$.

Image analysis. Standardized uptake values (SUV) were calculated as the tissue concentration of radioactivity $(\mathrm{kBq} / \mathrm{ml})$ divided by the injected dose per weight $(\mathrm{kBq} / \mathrm{g})$. To measure the maximum SUV $\left(\mathrm{SUV}_{\max }\right)$, a volume of interest was selected on integrated PET/CT images for tumor volume and metastatic LNs separately. Tumors were delineated using thresholds of $40,50,60,70$, and $80 \%$ of $\mathrm{SUV}_{\text {max }}$, and metabolic tumor volume and mean SUV ( $\left.\mathrm{SUV}_{\text {mean }}\right)$ were measured. Total lesion glycolysis was calculated by multiplying the $\mathrm{SUV}_{\text {mean }}$. The tumoral heterogeneity was represented by the heterogeneity factor (HF) (Figure 1). The tumor volume was determined with a series of SUV thresholds (40, 50, 60, 70 , and $80 \%$ of $\mathrm{SUV}_{\max }$ ). The HF was sorted from the linear regression line and calculated as the derivative $(\mathrm{dV} / \mathrm{dT})$ of the volume-threshold function (16). It is shown as a negative value.

Statistical analysis. Short-term outcome was assessed using both the treatment response evaluation by RECIST and the recurrence event during the follow-up. Consequently, treatment outcome was categorized into complete response by RECIST and no recurrence at the last follow-up (complete response/no recurrence group) and partial response, stable disease, or progressive disease by RECIST or recurrence during follow-up (residual disease/recurrence group). Disease-free survival was defined as the time between the completion of treatment and the first recurrence of the disease (local, regional, and distant recurrence).

We used the Mann-Whitney test to compare HFs across different clinical parameters. We used the chi-square test or Fisher's exact test to evaluate the response to induction chemotherapy or radiotherapy. Spearman rank correlation test was conducted to evaluate the correlation between HFs and other parameters of $\mathrm{PET} / \mathrm{CT}$. To evaluate locoregional recurrences, we performed 

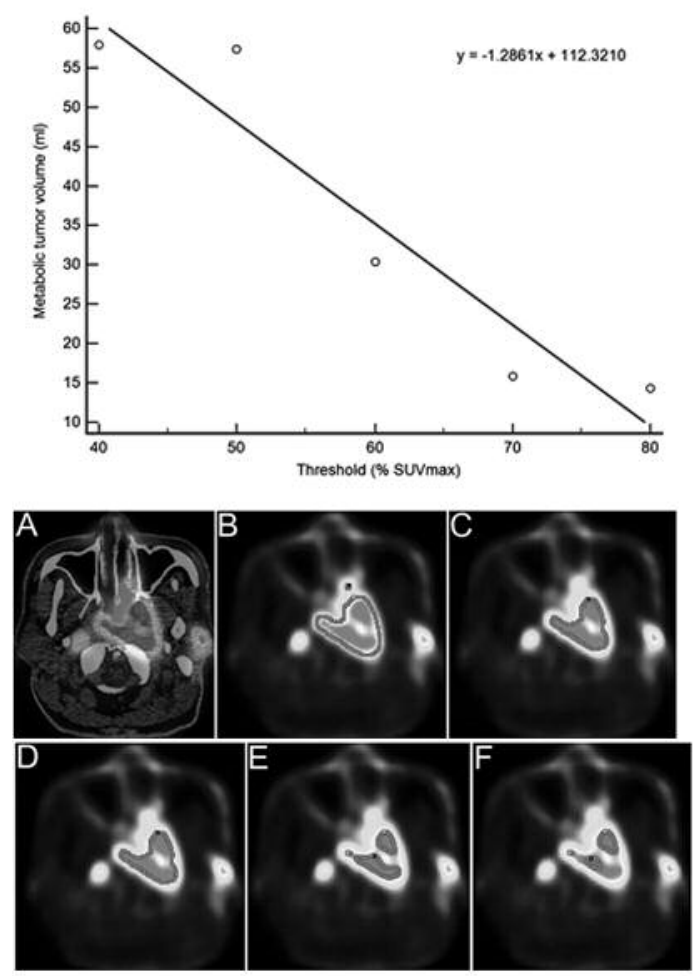

Figure 1. Graph shows representative volume-threshold function acquired by plotting thresholds to volume. Heterogeneity factor $(H F)$ was calculated by finding the derivative ( $d V / d T)$ of the volume-threshold function for each tumor (upper panel). Representative PET/CT images in nasopharyngeal cancer using several threshold methods. Decreasing tumor volumes are noted by $40 \%$ to $80 \%$ threshold of $S U V_{\max }$ (lower panel).

Kaplan-Meier analysis with the log-rank test. Software SPSS Version 18 (SPSS, Inc., Chicago, IL, USA) was used for the analyses. A $p$-value of less than 0.05 was considered statistically significant.

\section{Results}

Tumor heterogeneity factors are associated with clinical stages. Results of univariate analyses performed to evaluate the association between clinical parameters and HFs of primary tumors and metastatic LNs are shown in Table I. Age and sex were not significantly correlated with HFs of primary tumors and metastatic LNs. HFs of primary tumors were significantly high in hypopharyngeal cancers compared to those of other subsites of tumors, whereas HFs of metastatic LNs were not correlated with tumor subsite. HFs of primary tumors were significantly low in patients with advanced-stage cancers (Table I, T3-4 vs. T1-2, $p<0.001$; N2-3 vs. N0-1, $p=0.032$; Clinical stage III-IV versus stage I-II, $p=0.002$ ), whereas HFs of metastatic LNs were significantly low in patients with advanced nodal stage
Table I. Relationship between heterogeneity factors and clinical parameters.

\begin{tabular}{|c|c|c|c|}
\hline Characteristics & $\begin{array}{c}\text { Number } \\
(\text { Mean } \pm S D)\end{array}$ & $\mathrm{HF}$ & $p$-Value \\
\hline \multicolumn{4}{|c|}{$\mathrm{HF}$ of primary tumors $(\mathrm{pHF}), \mathrm{N}=52$} \\
\hline Age & & & 0.675 \\
\hline$<65$ & 31 & $-0.25 \pm 0.25$ & \\
\hline$\geq 65$ & 21 & $-0.22 \pm 0.28$ & \\
\hline Gender & & & 0.569 \\
\hline Male & 49 & $-0.24 \pm 0.27$ & \\
\hline Female & 3 & $-0.30 \pm 0.24$ & \\
\hline Tumor subsite & & & 0.014 \\
\hline Nasopharynx & 10 & $-0.34 \pm 0.36$ & \\
\hline Oropharnyx & 19 & $-0.31 \pm 0.30$ & \\
\hline Hypopharynx & 23 & $-0.14 \pm 0.11$ & \\
\hline Clinical $\mathrm{T}$ classification & & & $<0.001$ \\
\hline $\mathrm{T} 1-2$ & 33 & $-0.14 \pm 0.11$ & \\
\hline $\mathrm{T} 3-4$ & 19 & $-0.41 \pm 0.35$ & \\
\hline Clinical $\mathrm{N}$ classification & & & 0.032 \\
\hline N0-1 & 16 & $-0.15 \pm 0.13$ & \\
\hline $\mathrm{N} 2-3$ & 36 & $-0.28 \pm 0.30$ & \\
\hline Clinical stage & & & 0.002 \\
\hline Stage I-II & 10 & $-0.09 \pm 0.07$ & \\
\hline Stage III-IV & 42 & $-0.28 \pm 0.28$ & \\
\hline \multicolumn{4}{|c|}{$\begin{array}{l}\text { HF of metastatic lymph nodes } \\
(\mathrm{nHF}), \mathrm{N}=41\end{array}$} \\
\hline Age & & & 0.813 \\
\hline$<65$ & 23 & $-0.27 \pm 0.27$ & \\
\hline$\geq 65$ & 18 & $-0.21 \pm 0.15$ & \\
\hline Sex & & & 0.423 \\
\hline Male & 38 & $-0.25 \pm 0.23$ & \\
\hline Female & 3 & $-0.13 \pm 0.06$ & \\
\hline Tumor subsite & & & 0.558 \\
\hline Nasopharynx & 9 & $-0.35 \pm 0.32$ & \\
\hline Oropharynx & 14 & $-0.23 \pm 0.22$ & \\
\hline Hypopharynx & 18 & $-0.20 \pm 0.15$ & \\
\hline Clinical $\mathrm{T}$ classification & & & 0.217 \\
\hline $\mathrm{T} 1-2$ & 23 & $-0.20 \pm 0.16$ & \\
\hline T3-4 & 18 & $-0.30 \pm 0.28$ & \\
\hline Clinical N classification & & & 0.028 \\
\hline N0-1 & 6 & $-0.10 \pm 0.07$ & \\
\hline $\mathrm{N} 2-3$ & 35 & $-0.27 \pm 0.23$ & \\
\hline Clinical stage & & & 0.028 \\
\hline Stage I-II & 3 & $-0.06 \pm 0.07$ & \\
\hline Stage III-IV & 38 & $-0.26 \pm 0.22$ & \\
\hline
\end{tabular}

HF, Heterogeneity factor; SD, standard deviation. Bold numbers indicate statistical significance $(p<0.05)$.

cancers (Table I, N2-3 vs. N0-1, $p=0.028$; Clinical stage IIIVI vs. stage I-II, $p=0.028)$. Clinical $\mathrm{T}$ stages were not associated with HFs of metastatic LNs $(p=0.217)$.

Tumor HFs of primary tumors, but not of metastatic LNs, are associated with other PET/CT parameters. $\mathrm{SUV}_{\max }$ was not significantly correlated with HFs of primary tumors and metastatic LNs (Figure 2A and D). Metabolic tumor volume 
A
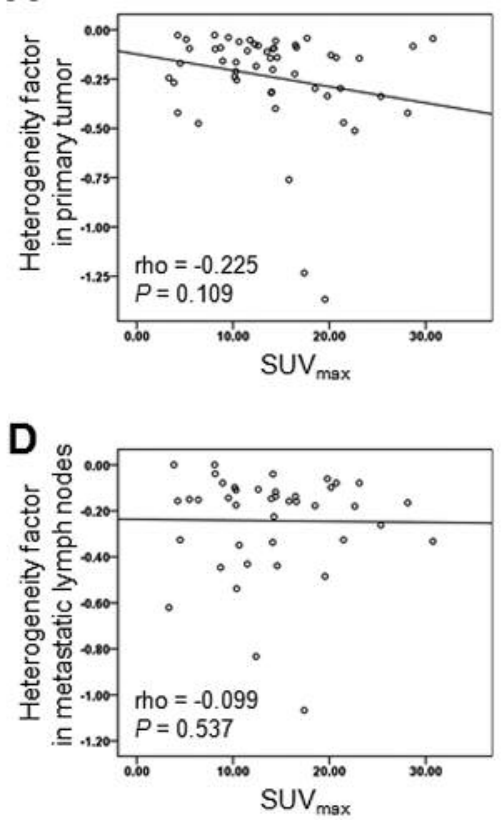

B

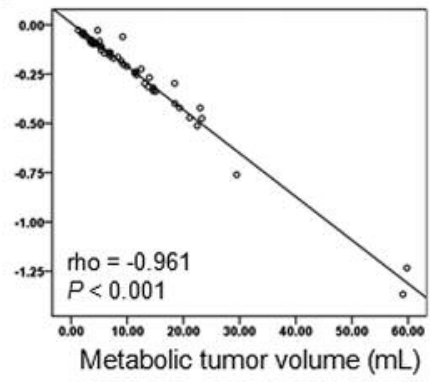

E

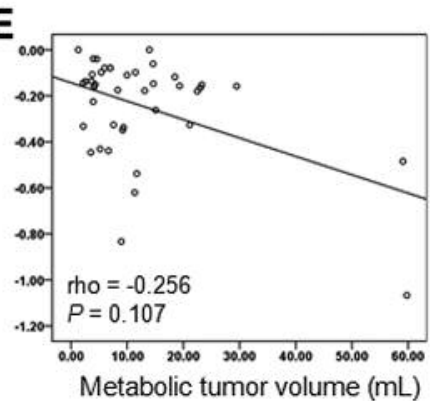

C

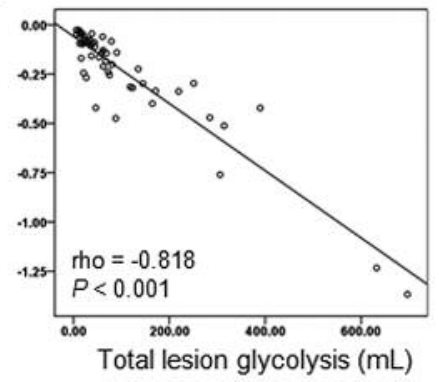

$\mathbf{F}$

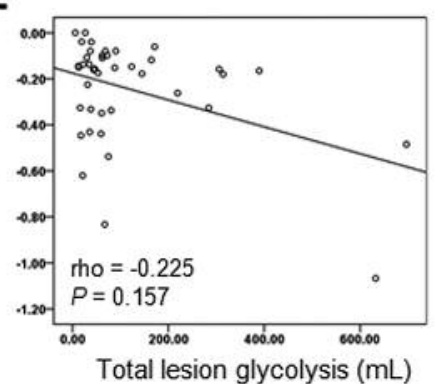

Figure 2. Correlation between heterogeneity factors and other PET/CT variables including SUVmax (A and D), metabolic tumor volume (B and E), and total lesion glycolysis ( $C$ and $F$ ).

and total lesion glycolysis showed statistically significant and strong associations with HFs of primary tumors (Figure $2 \mathrm{~B}-\mathrm{C}$, rho $=-0.961, p<0.001$ for metabolic tumor volume, rho $=-0.818, p<0.001$ for total lesion glycolysis). However, HFs of metastatic LNs were not significantly associated with metabolic tumor volume and total lesion glycolysis (Figure 2E-F). Analyses of associations between HFs of primary tumors and those in metastatic LNs revealed no significant association between the two parameters (Figure 3, rho $=0.226, p=0.156)$.

HFs of primary tumors, but not of metastatic LNs, are correlated with response to radiotherapy and are predictive factors for locoregional recurrences. We assessed the treatment response in primary tumors and LNs after induction chemotherapy or radiotherapy (Table II). The patients were divided into a high HF group and a low HF group according to the criteria of median values of HFs (median value of primary tumor $\mathrm{HFs}=-0.15$, median value of metastatic LN HFs=-0.16). Consequently, HFs of primary tumors and metastatic LNs were not significantly associated with treatment response after induction chemotherapy. Importantly, response of primary tumors to radiotherapy was significantly lower in patients with low HFs of primary tumors (Table II, 92.3\% [24/26] of patients experienced complete remission in the high HF group versus $69.2 \%$

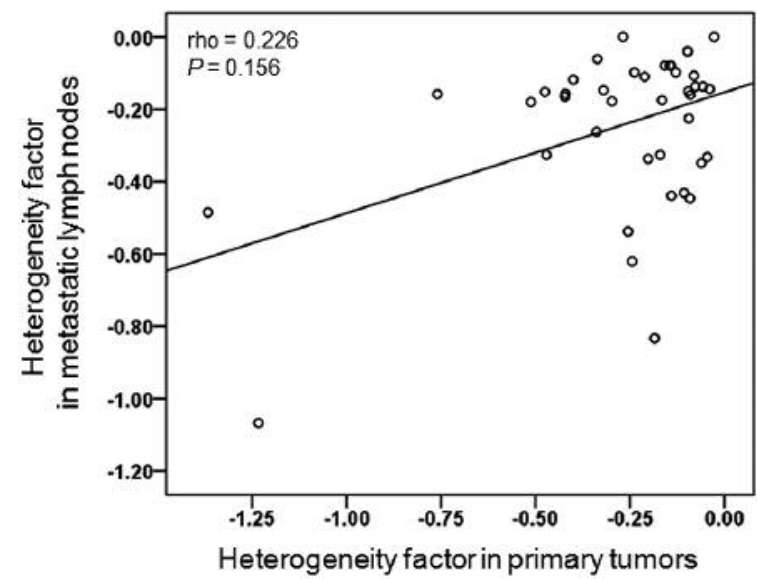

Figure 3. Correlation between heterogeneity factors in primary tumors and those in metastatic lymph nodes.

[18/26] in the low HF group, $p=0.035)$, whereas response of LNs was not correlated with HFs of primary tumors. HFs of metastatic LNs were not associated with response to radiotherapy in both primary tumors and LNs.

We further performed Kaplan-Meier analyses to evaluate locoregional recurrences after treatment. The patients with 
A

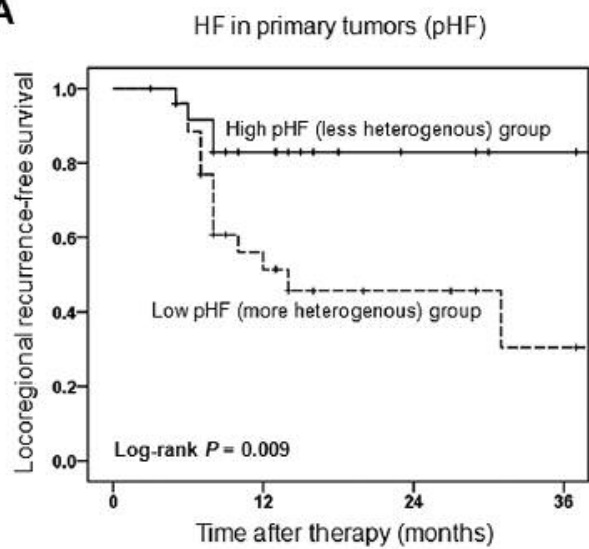

B $\quad$ HF in metastatic lymph nodes (nHF)

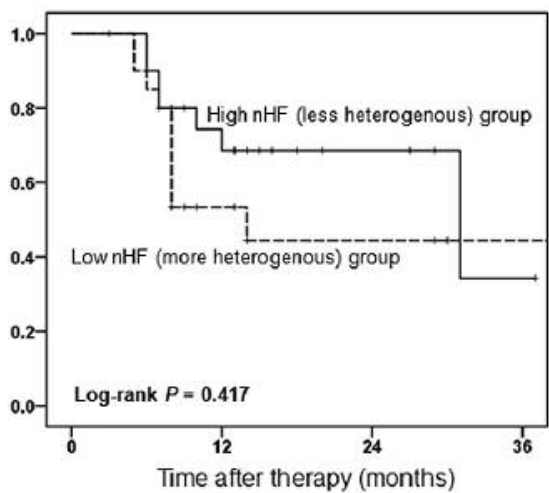

Figure 4. (A) Locoregional recurrence-free survival according to heterogeneity factors in primary tumor (pHF). (B) Locoregional recurrence-free survival according to heterogeneity factors in metastatic lymph nodes $(\mathrm{nHF})$.

Table II. Response to induction chemotherapy and radiotherapy according to heterogeneity factors.

\begin{tabular}{|c|c|c|c|c|c|c|}
\hline \multirow[b]{2}{*}{ Treatment response } & \multicolumn{3}{|c|}{ pHF (median -0.15 ) } & \multicolumn{3}{|c|}{ nHF (median -0.16 ) } \\
\hline & $\begin{array}{c}\text { High HF } \\
\text { N }(\%)\end{array}$ & $\begin{array}{c}\text { Low HF } \\
\mathrm{N}(\%)\end{array}$ & $p$-Value & $\begin{array}{c}\text { High HF } \\
\mathrm{N}(\%)\end{array}$ & $\begin{array}{c}\text { Low HF } \\
\mathrm{N}(\%)\end{array}$ & $p$-Value \\
\hline \multicolumn{7}{|l|}{ Response to IC } \\
\hline Primary tumor & & & 1.000 & & & 1.000 \\
\hline $\mathrm{CR} / \mathrm{PR}$ & $17(94.4)$ & $20(90.9)$ & & $14(93.3)$ & $16(94.1)$ & \\
\hline $\mathrm{SD} / \mathrm{PD}$ & $1(5.6)$ & $2(9.1)$ & & $1(6.7)$ & $1(5.9)$ & \\
\hline Lymph nodes & & & 0.427 & & & 0.659 \\
\hline $\mathrm{CR} / \mathrm{PR}$ & $16(88.9)$ & $17(77.3)$ & & $13(86.7)$ & $13(76.5)$ & \\
\hline $\mathrm{SD} / \mathrm{PD}$ & $2(11.1)$ & $5(22.7)$ & & $2(13.3)$ & $4(23.5)$ & \\
\hline \multicolumn{7}{|l|}{ Response to RT } \\
\hline Primary tumor & & & 0.035 & & & 0.277 \\
\hline $\mathrm{CR}$ & $24(92.3)$ & $18(69.2)$ & & $18(85.7)$ & $14(70.0)$ & \\
\hline $\mathrm{PR} / \mathrm{SD} / \mathrm{PD}$ & $2(7.7)$ & $8(30.8)$ & & $3(14.3)$ & $6(30.0)$ & \\
\hline Lymph nodes & & & 0.109 & & & 0.265 \\
\hline $\mathrm{CR}$ & $22(84.6)$ & $17(65.4)$ & & $16(76.2)$ & $12(60.0)$ & \\
\hline $\mathrm{PR} / \mathrm{SD} / \mathrm{PD}$ & $4(15.4)$ & $9(34.6)$ & & $5(23.8)$ & $8(40.0)$ & \\
\hline
\end{tabular}

pHF, Heterogeneity factor in primary tumor; nHF, heterogeneity factor in metastatic lymph nodes; IC, induction chemotherapy; RT, radiotherapy, $\mathrm{CR}$, complete remission; PR, partial remission; SD, stable disease; PD, progressive disease. Bold numbers indicate statistical significance $(p<0.05)$.

low HFs of primary tumors showed a lower locoregional recurrence-free survival rate than those with high $\mathrm{HFs}$ of primary tumors did (Figure 4A, 2-year locoregional recurrence-free survival rates of $82.9 \%$ in the high $\mathrm{pHF}$ group $v s .45 .7 \%$ in the low pHF group, log-rank $p=0.009$ ). HFs of metastatic LNs were not significantly predictive of locoregional recurrence (Figure 4B, 2-year locoregional recurrence-free survival rates of $68.6 \%$ in the high $\mathrm{nHF}$ group $v s .44 .4 \%$ in the low nHF group, log-rank $p=0.417$ ).

\section{Discussion}

In the present study, we measured HFs of primary tumor and metastatic LNs from PET/CT images, and evaluated their clinical significance in parallel. The results showed that HFs of primary tumors, but not of metastatic LNs, correlated with other prognostic parameters and were significant predictors of responses to radiotherapy and locoregional recurrences. 
Recently, HFs of PET/CT, defined as the derivative $(\mathrm{dV} / \mathrm{dT})$ of a volume-threshold function, have been reported to be a clinically useful indicator in head and neck cancers. For example, intratumoral heterogeneity has been suggested as a significant prognostic factor in oral cavity, nasopharyngeal, and sinonasal cancer $(14,17,18)$. The HF has also been utilized as a reliable and non-invasive method for differentiation of malignant and benign parotid gland tumors(16). Consistent with these results, our study demonstrated that the HFs of primary tumors were significantly predictors of response to radiotherapy and locoregional recurrences in pharyngeal cancers treated with chemoradiotherapy. Thus, pHF could be a useful prognostic parameter in pharyngeal cancer, and other head and neck cancer subtypes.

In case of pharyngeal cancers, tumor burdens of metastatic LNs are frequently high despite the small primary tumor sizes. Advanced metastatic LNs contribute to the failure of radiotherapy. On the basis of these unique characteristics of pharyngeal cancers, we initially hypothesized that the metabolic heterogeneity of metastatic LNs might be a predictor of outcomes in pharyngeal cancers. However, our results indicate that the HFs of metastatic LNs are not predictive of response to radiotherapy and the 2-year locoregional recurrence rate, whereas the HFs of primary tumors were significantly correlated with the prognoses. The reason why the HFs of metastatic LNs were not correlated with prognosis is not yet known. However, the differences in tumor biology between primary tumors and metastatic LNs may have resulted in the lack of correlation between metabolic heterogeneity and prognosis.

This work is significant because it provides a novel predictive factor for 2-year treatment outcomes in patients with pharyngeal cancers treated with chemoradiotherapy. Although chemoradiation provide acceptable oncological outcomes while minimizing morbidity, treatment failure often elicits devastating results including progressive disease requiring multiple salvage operations. For patients designated as potential poor responders to chemoradiation, such as those with T4a-stage-hypopharyngeal cancers, upfront surgery with adjuvant therapy might improve treatment outcomes. It is important to determine patient responsiveness prior to definitive chemoradiation. Out data identify the HFs of primary tumors as clinically useful indicators that are predictive of response to radiotherapy and 2-year locoregional recurrence in pharyngeal cancer. The use of HF as a predictive factor will allow an optimal selection of treatment strategies and prevent unnecessary treatment and delays in surgical intervention in a significant number of patients.

This study has several limitations including its retrospective nature and the potential for selection bias. Also, a small number of patients $(\mathrm{n}=52)$ patients were enrolled. Further large-scale prospective evaluations will be required to resolve these deficiencies. Moreover, the primary tumor
HF was well correlated with other important prognostic factors such as tumor stage and volume in this study, suggesting that the primary tumor HF should be considered only in conjunction with prognosticators and not as an independent prognostic factor. Although the HF was found to be correlated with other parameters such as $\mathrm{SUV}_{\max }$, metabolic tumor volume, and total lesion glycolysis, it is only one of many potential metabolism parameters and is not fully representative of tumor heterogeneity. For a more detailed evaluation of radiological heterogeneity, textural parameters such as coarseness, busyness, complexity, and contrast must be investigated (19).

In conclusion, HFs of primary tumors and metastatic LNs have differential associations with prognosis in patients with pharyngeal cancers treated with chemoradiotherapy. The HFs of primary tumors were significant predictors of response to radiotherapy and prognostic factors for locoregional recurrence in pharyngeal cancer, whereas the HFs of metastatic LNs were not significant prognostic factors. The use of $\mathrm{HF}$ as a predictive factor will aid clinicians in selecting the best treatment strategies, and prevent unnecessary treatment and avoidable delays in surgical intervention in patients with pharyngeal cancer.

\section{Funding}

This study was supported by Biomedical Research Institute Grant (2015-42), Pusan National University Hospital.

\section{Conflicts of Interest}

The Authors declare that no conflict of interest exists.

\section{References}

1 Belcher R, Hayes K, Fedewa S and Chen AY: Current treatment of head and neck squamous cell cancer. J Surg Oncol 110: 551574, 2014.

2 Hoch S, Thelen K, Vorwerk H, Netzer C, Wilhelm T, Günzel T and Teymoortash A: Impact of different treatment concepts on regional failure in advanced oropharyngeal cancer. Anticancer Res 37(2): 727-734, 2017.

3 Rios Velazquez E, Hoebers F, Aerts HJ, Rietbergen MM, Brakenhoff RH, Leemans RC, Speel EJ, Straetmans J, Kremer B and Lambin P: Externally validated HPV-based prognostic nomogram for oropharyngeal carcinoma patients yields more accurate predictions than TNM staging. Radiother Oncol 113: 324-330, 2014.

4 Ribeiro DA, Nascimento FD, Fracalossi AC, Noguti J, Oshima CT, Ihara SS and Franco MF: The role of metalloendopeptidases in oropharyngeal carcinomas assessed by tissue microarray. Cancer Genomics Proteomics 8(6): 307-310, 2011.

5 Ribeiro DA, Nascimento FD, Fracalossi AC, Gomes TS, Oshima CT and Franco MF: Expression profiling of cell cycle regulatory proteins in oropharyngeal carcinomas using tissue microarrays. In Vivo 24(3): 315-320, 2010. 
6 Higgins KA, Hoang JK, Roach MC, Chino J, Yoo DS, Turkington TG and Brizel DM: Analysis of pretreatment FDGPET SUV parameters in head-and-neck cancer: tumor SUVmean has superior prognostic value. Int J Radiat Oncol Biol Phys 82(2): 548-553, 2012.

7 Suzuki H, Kato K, Fujimoto Y, Itoh Y, Hiramatsu M, Naganawa S, Hasegawa Y and Nakashima T: Prognostic value of (18)Ffluorodeoxyglucose uptake before treatment for pharyngeal cancer. Ann Nucl Med 28(4): 356-362, 2014.

8 Chen SW1, Hsieh TC, Yen KY, Liang JA and Kao CH: Pretreatment (18)F-FDG PET/CT in whole-body total lesion glycolysis to predict survival in patients with pharyngeal cancer treated with definitive radiotherapy. Clin Nucl Med 39: e296300, 2014.

9 Pak K, Cheon GJ, Nam HY Kim SJ, Kang KW, Chung JK, Kim EE and Lee DS: Prognostic value of metabolic tumor volume and total lesion glycolysis in head and neck cancer: a systematic review and meta-analysis. J Nucl Med 55: 884-890, 2014.

10 Mroz EA, Tward AD, Hammon RJ, Ren Y and Rocco JW: Intratumor genetic heterogeneity and mortality in head and neck cancer: analysis of data from the Cancer Genome Atlas. PLoS med 12: e1001786, 2015.

11 Mroz EA, Tward AD, Pickering CR, Myers JN, Ferris RL and Rocco JW: High intratumor genetic heterogeneity is related to worse outcome in patients with head and neck squamous cell carcinoma. Cancer 119: 3034-3042, 2013.

12 Nakajima EC, Laymon C, Oborski M, Hou W, Wang L, Grandis JR, Ferris RL, Mountz JM and Van Houten B: Quantifying metabolic heterogeneity in head and neck tumors in real time: 2-DG uptake is highest in hypoxic tumor regions. PloS one 9: e102452, 2014.

13 Salamon J1, Derlin T, Bannas P, Busch JD, Herrmann J, Bockhorn M, Hagel C, Friedrich RE, Adam G and Mautner VF: Evaluation of intratumoural heterogeneity on ${ }^{18} \mathrm{~F}-\mathrm{FDG} \mathrm{PET} / \mathrm{CT}$ for characterization of peripheral nerve sheath tumours in neurofibromatosis type 1. Eur J Nucl Med Mol Imaging 40: 685$692,2013$.
14 Huang B1, Chan T, Kwong DL, Chan WK and Khong PL: Nasopharyngeal carcinoma: investigation of intratumoral heterogeneity with FDG PET/CT. AJR Am J Roentgenol 199: 169-174, 2012.

15 Bundschuh RA, Dinges J, Neumann L, Seyfried M, Zsótér N, Papp L, Rosenberg R, Becker K, Astner ST, Henninger M, Herrmann K, Ziegler SI, Schwaiger M and Essler M: Textural Parameters of Tumor Heterogeneity in ${ }^{18}$ F-FDG PET/CT for Therapy Response Assessment and Prognosis in Patients with Locally Advanced Rectal Cancer. J Nucl Med 55: 891-897, 2014.

16 Kim BS, Kim SJ and Pak K: Diagnostic value of metabolic heterogeneity as a reliable parameter for differentiating malignant parotid gland tumors. Ann Nucl Med 30: 346-354, 2016.

17 Kwon SH1, Yoon JK, An YS, Shin YS, Kim CH, Lee DH, Jo $\mathrm{KS}$ and Lee SJ: Prognostic significance of the intratumoral heterogeneity of (18) F-FDG uptake in oral cavity cancer. J Surg Oncol 110: 702-706, 2014.

18 Kim BS, Pak K, Yi KI, Kim IJ, Roh HJ and Cho KS: Prognostic value of tumoral heterogeneity and volumetric parameters as measured by F18-FDG PET/CT in sinonasal cancer. Eur Arch Otorhinolaryngol 274(3): 1437-1443, 2017.

19 Oh JS, Kang BC, Roh JL, Kim JS, Cho KJ, Lee SW, Kim SB, Choi SH, Nam SY and Kim SY: Intratumor textural heterogeneity on pretreatment (18)F-FDG PET images predicts response and survival after chemoradiotherapy for hypopharyngeal cancer. Ann Surg Oncol 22: 2746-2754, 2015.

Received August 4, 2017

Revised September 5, 2017

Accepted September 6, 2017 